DRAFT VERSION SEPTEMBER 11, 2021

Preprint typeset using LTEX style emulateapj v. 08/17/12

\title{
MEASUREMENTS OF NON-THERMAL LINE WIDTHS IN SOLAR ACTIVE REGIONS
}

\author{
DAVID H. BROOKS ${ }^{1,3}$ AND HARRY P. WARREN ${ }^{2}$ \\ ${ }^{1}$ College of Science, George Mason University, 4400 University Drive, Fairfax, VA 22030 USA and \\ ${ }^{2}$ Space Science Division, Naval Research Laboratory, Washington, DC 20375 USA \\ Draft version September 11, 2021
}

\begin{abstract}
Spectral line widths are often observed to be larger than can be accounted for by thermal and instrumental broadening alone. This excess broadening is a key observational constraint for both nanoflare and wave dissipation models of coronal heating. Here we present a survey of non-thermal velocities measured in the high temperature loops (1-4 MK) often found in the cores of solar active regions. This survey of Hinode Extreme Ultraviolet Imaging Spectrometer (EIS) observations covers 15 non-flaring active regions that span a wide range of solar conditions. We find relatively small non-thermal velocities, with a mean value of $17 \mathrm{~km} \mathrm{~s}^{-1}$, and no significant trend with temperature or active region magnetic flux. These measurements appear to be inconsistent with those expected from reconnection jets in the corona, chromospheric evaporation induced by coronal nanoflares, and Alfvén wave turbulence models. Furthermore, because the observed non-thermal widths are generally small, such measurements are difficult and susceptible to systematic effects.
\end{abstract}

Subject headings: Sun: corona-Sun: UV radiation-methods: data analysis

\section{INTRODUCTION}

The solution to the problem of how the solar corona maintains its high temperature relative to the cool solar photosphere is still unresolved. There have been many explanations put forward, and there are several reviews on the topic in the literature from varying perspectives (e.g. Mandrini et al. 2000; Walsh \& Ireland 2003; Klimchuk 2006; Reale 2010; Parnell \& De Moortel 2012; Arreguil 2015). Two of the most studied ideas are based on magnetic reconnection and MHD waves. In the reconnection scenario, the magnetic field in the corona is braided by turbulent convection in the photosphere, and the energy is released through small-scale events popularly known as nanoflares (Parker 1983, 1988). In the MHD wave scenario, the interaction between photospheric convection and the magnetic field can produce waves that propagate upward along the magnetic field and dissipate energy through a variety of possible mechanisms such as phase mixing (Hevvaerts \& Priest 1983), or resonant absorption (Ionson 1978). More recently, chromospheric jets have been suggested as a mechanism for supplying mass and energy to the corona directly from the lower atmospheric layers (De Pontieu et al. 2009, 2011).

There has been tentative observational evidence for several of the predicted details of these models. De Pontieu et al. (2011), for instance, show examples of chromospheric spicules producing an apparent response at transition region and coronal temperatures. Cirtain et al. (2013), show cases of large scale wrapped and twisted coronal structures that could be evidence of magnetic braiding. Testa et al. (2013) find evidence of rapid variability at the footpoints of hot loops that they interpret as signatures of heating associated with reconnection events occuring in the overlying loops. Very recently, Okamoto et al. (2015) observed decreasing amplitude wave-like motions in chromospheric images that they suggest are evidence of resonant absorption. Future observations and comparisons with numerical models will clarify if these interpretations are correct.

\footnotetext{
${ }^{3}$ Present address: Hinode Team, ISAS/JAXA, 3-1-1 Yoshinodai, Chuoku, Sagamihara, Kanagawa 252-5210, Japan
}

Despite these recent advances, and we have highlighted only a few examples, in general it has been difficult to discriminate between the theoretical models and reconcile them with all aspects of the observations. One diagnostic that has the potential to be a good discriminator is the measurement of non-thermal broadening in excess of the thermal and instrumental broadening of EUV spectral lines. As pointed out by Cargill (1996), the nanoflare-heated corona model predicts the existence of many reconnection jets in multiple directions along an observed line-of-sight, and this should lead to significant non-thermal velocities on the order of $250 \mathrm{~km}$ $\mathrm{s}^{-1}$ or larger. In contrast, hydrodynamic models of chromospheric evaporation in response to coronal nanoflares suggest non-thermal velocities of $20-36 \mathrm{~km} \mathrm{~s}^{-1}$, increasing with temperature, at coronal loop tops in the 1.1-5.6 MK temperature range (Patsourakos \& Klimchuk 2006). Models of shock heating driven by Alfvén waves also predict high velocities, $>100 \mathrm{~km} \mathrm{~s}^{-1}$, see e.g. Antolin et al. (2008), while models of Alfvén wave turbulence (van Ballegooijen et al. 2011) show non-thermal velocities of $25-35 \mathrm{~km} \mathrm{~s}^{-1}$ at the tops of loops formed near 1.6 MK (Asgari-Targhi et al. 2014). Furthermore, models that attempt to explain the first ionization potential (FIP) effect based on the forces acting on propagating waves suggest velocities on the order of $50-80 \mathrm{~km} \mathrm{~s}^{-1}$ (Laming 2004, 2012).

These are significant differences that are, in principal, observationally detectable, so that at least some of the possible explanations for the observed non-thermal broadening could be ruled out, even if the heating mechanism itself cannot be verified. For example, measurements of non-thermal velocities of $40 \mathrm{~km} \mathrm{~s}^{-1}$ or so would tend to support a chromospheric evaporation or Alfvén wave turbulence explanation, though clearly they could not discriminate between them. Conversely, the nanoflare model may predict high non-thermal velocities, but these may only be apparent immediately after the energy release, and could be difficult to detect due to the low emission measure of any hot plasma, a problem that may be further exacerbated by non-equilibrium conditions (Bradshaw \& Cargill 2006).

The presence of a hot active region (AR) plasma component 
is in fact a central prediction of impulsive heating models, and significant effort has been expended assessing whether such plasma can be observed. See e.g. Reale et al. (2009), Reale et al. (2009), and Schmelz et al. (2009) for initial reports of detections, Reale (2014) for a review of subsequent efforts, and O'Dwyer et al. (2011) and Winebarger et al. (2012) for reports of negative results and the difficulties of observing the appropriate temperature range. Notwithstanding these problems, measurements of non-thermal velocities at high temperatures (and any trend with temperature) are clearly significant for establishing whether the nanoflareheated corona model is viable.

Previous measurements, however, have indicated that the excess width of EUV and soft X-ray lines is smaller than suggested by Cargill (1996). Early observations from Skylab indicated values of $10-25 \mathrm{~km} \mathrm{~s}^{-1}$ for UV coronal lines formed near 1.7 MK such as Fe XI and Fe XII, for example, see e.g. Cheng et al. (1979). Later measurements of the $\mathrm{Mg}$ XI resonance line formed around 2.8 MK from the Solar Maximum Mission were found to be somewhat larger: 40-60 km s ${ }^{-1}$ (Acton et al. 1981; Saba \& Strong 1991), but these are also significantly smaller than expected from the high temperature reconnection jets. Recent studies using Hinode/EIS (EUV Imaging Spectrometer, Culhane et al. 2007) and SOHO/SUMER (Solar Ultraviolet Measurements of Emitted Radiation, Wilhelm et al. 1995) have succeeded in observing hot plasma emission from the Ca XVII $192.858 \AA$ and Fe XVIII 974.86 $\AA$ lines formed near 5.6 MK and 7.1 MK, respectively (Ko et al. 2009; Teriaca et al. 2012). They did not, however, assess the non-thermal broadening, and it is difficult for solar observations to access much higher temperatures outside of flares.

Observations of stellar atmospheres, however, have routinely been made in the 7-11 MK range with high spectral resolution instruments on space missions such as the Far Ultraviolet Spectroscopic Explorer (FUSE) and the Hubble Space Telescope. Redfield et al. (2003) have studied the profiles of the Fe XVIII $974 \AA$ and Fe XIX $1119 \AA$ lines, formed around 7.1 MK and $8.9 \mathrm{MK}$, for a sample of late type stars observed by FUSE. Linsky et al. (1998) used the HST Goddard High Resolution Spectrograph to examine the profile of the Fe XXI $1354 \AA$ line, formed near $11.2 \mathrm{MK}$, in the atmosphere of the Capella binary. Both of these studies basically found no appreciable non-thermal line broadening and concluded that all these lines were consistent with thermal broadening.

Stellar observations of course have no spatial resolution, and that is the advantage of spectrometers such as EIS that have allowed the measurement of non-thermal velocities in discrete solar coronal structures. EIS observations have shown that the largest non-thermal velocities in an AR are located in the regions of lowest intensity (Del Zanna 2008; Doschek et al. 2008; Harra et al. 2008; Baker et al. 2009), where the plasma is outflowing and may contribute to the solar wind (Sakao et al. 2007; Harra et al. 2008; Doschek et al. 2008; Brooks et al. 2015). Measurements of the non-thermal velocities in these regions are about $30-60 \mathrm{~km} \mathrm{~s}^{-1}$ using lines formed at 1.6-2.2 MK (Del Zanna 2008; Doschek et al. 2008; Brooks \& Warren 2011), and these values are somewhat larger than observed in the AR core. Warren et al. (2008a) and Brooks \& Warren (2009), for example, obtained values of $20-30 \mathrm{~km} \mathrm{~s}^{-1}$ in the moss at the footpoints of 1.6 MK loops.

Despite these studies, there are relatively few accurate mea-
TABLE 1

ACtive Region OBSERVATIONS

\begin{tabular}{rccccc}
\hline \hline$\#$ & EIS Dataset & Slit $^{\mathrm{a}}$ & FOV $^{\mathrm{a}}$ & Exp.Time $^{\mathrm{b}}$ & Study ID $^{2}$ \\
\hline 1 & eis_11_20100619_014433 & 2 & 100x240 & 30 & 241 \\
2 & eis_11_20100621_011541 & 1 & 120x512 & 60 & 420 \\
3 & eis_11_20100723_143210 & 1 & $120 \times 512$ & 60 & 420 \\
4 & eis_11_20100929_223226 & 1 & $300 \times 400$ & 30 & 356 \\
5 & eis_11_20110121_133954 & 1 & $120 \times 512$ & 60 & 420 \\
6 & eis_11_20110131_102326 & 1 & $240 \times 512$ & 60 & 437 \\
7 & eis_11_20110212_143019 & 1 & $240 \times 512$ & 60 & 437 \\
8 & eis_11_20110411_105848 & 1 & $240 \times 512$ & 60 & 437 \\
9 & eis_11_20110415_001526 & 1 & $240 \times 512$ & 60 & 437 \\
10 & eis_11_20110419_123027 & 1 & $240 \times 512$ & 60 & 437 \\
11 & eis_11_20110702_030712 & 1 & $120 \times 512$ & 60 & 420 \\
12 & eis_11_20110725_090513 & 1 & $120 \times 512$ & 60 & 420 \\
13 & eis_11_20110821_105251 & 1 & $360 \times 512$ & 60 & 471 \\
14 & eis_11_20111108_181234 & 1 & $240 \times 512$ & 60 & 437 \\
15 & eis_11_20111110_100028 & 1 & $360 \times 512$ & 60 & 471 \\
\hline
\end{tabular}

${ }^{a}$ Units of arcseconds.

${ }^{\mathrm{b}}$ Units are seconds.

surements of non-thermal velocities in AR core loops. One of the few cases is that of Imada et al. (2009), who performed a very interesting study of an AR core at the limb, and reported an average value of $13 \mathrm{~km} \mathrm{~s}^{-1}$ at $2.8 \mathrm{MK}$. They studied the AR core as a whole, however, and did not isolate the emission from the high temperature loops, or investigate any trend with temperature in detail. Since the region was also observed at the limb, the mass motions may be diminished due to the line-of-sight. This effect was noted by Hara et al. (2008a), who found a decrease in the non-thermal velocities at the footpoints of $\sim 2 \mathrm{MK}$ loops as the AR they studied tracked from disk center to limb, a trend that has been suggested to be indicative of Alfvén wave heating (McClements et al. 1991; Cargill 1996). It would also be interesting to know if the AR studied by Imada et al. (2009) is typical or not.

Here we undertake a systematic survey of the non-thermal velocities in the hot plasma in AR cores, to assess whether the observations and coronal heating models can be reconciled. We isolate 16 loops in 15 different ARs covering a wide range of solar conditions, and measure the non-thermal velocities over a range of temperatures from 1.1-3.6 MK. We consider the magnitudes of the measurements, and any trend with temperature, both of which are important for nanoflare heating models.

\section{OBSERVATIONS}

For this study of spectral line widths, we use observations from EIS on Hinode (Kosugi et al. 2007). EIS is a normal incidence spectrograph with multi-layer coatings on the mirror and grating. It observes the solar EUV spectrum in two wavelength channels: a short-wavelength (SW) channel covering 170-210 $\AA$, and a long-wavelength (LW) channel covering $250-290 \AA$. This part of the spectrum is dominated by emission lines from the Fe VII-Fe XVII ionization stages of Iron, with numerous other lines from other elements. These lines permit a very broad temperature coverage of observed solar features, and many of the lines are also clean and relatively unblended, making them ideal for our purpose. Lists of EIS spectral line identifications have been compiled by Young et al. (2007) and Brown et al. (2008).

EIS builds raster scan spectral images by stepping its slit across an area of the Sun using a scan mirror. There are four slits: $1^{\prime \prime}, 2^{\prime \prime}, 40^{\prime \prime}$, and $266^{\prime \prime}$, with the narrowest $1^{\prime \prime}$ slit pro- 

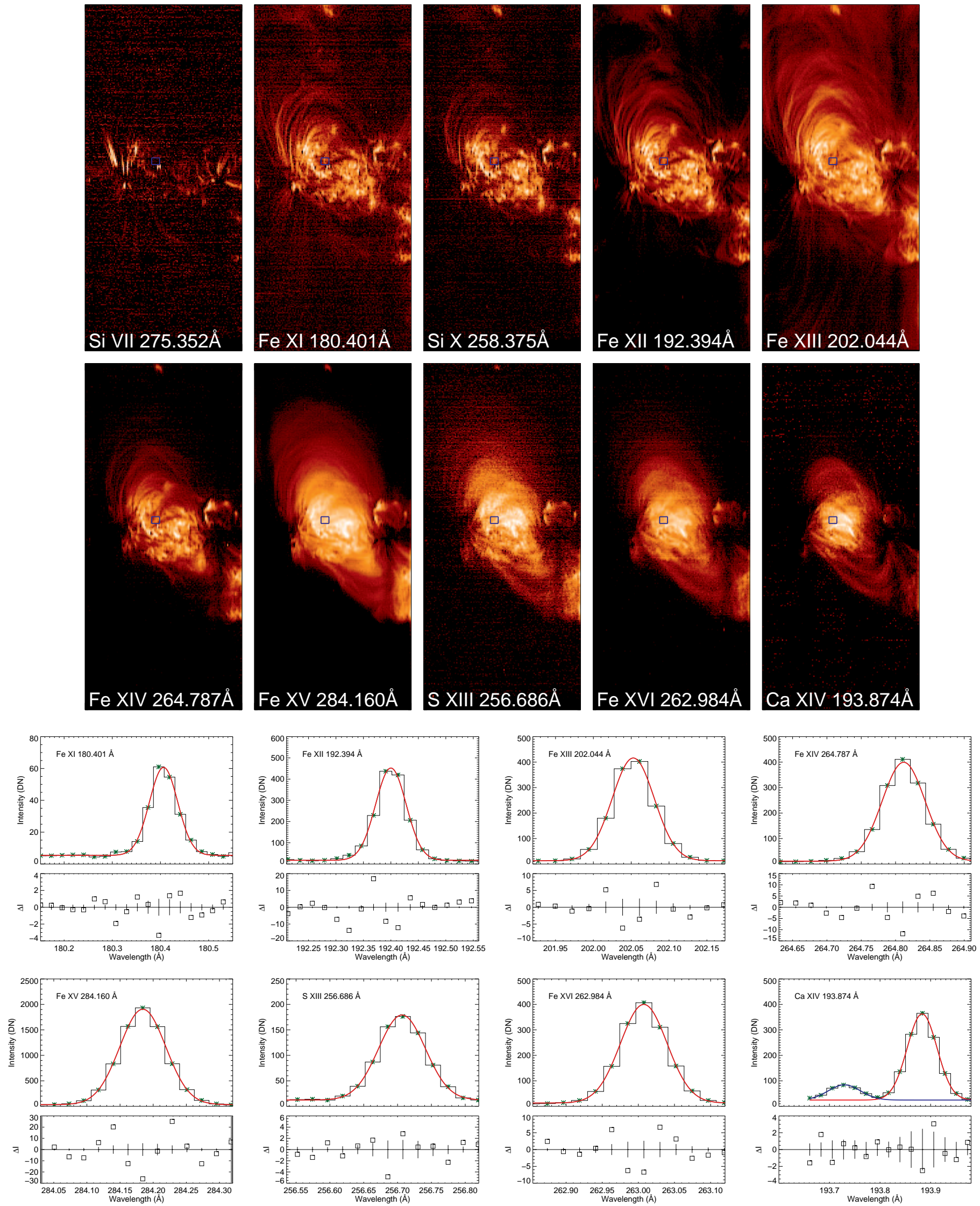

FIG. 1. - Example spectral images of AR 1193 observed on 2011, April 19. We measured the line widths in the high temperature core loops for the region indicated by the blue box. Example fits for several of the spectral lines are shown in the lower panels. 
viding the best spectral resolution, which is about $22 \mathrm{~m} \AA$ for 1 EIS pixel. To obtain the most accurate line width measurements, we mostly use the highest spectral resolution slit in this study (14/15 datasets). The choice of slit, field-of-view (FOV) of the raster scan, spectral line list, exposure time etc. are fixed when the EIS observing study is defined. We use data from five different EIS studies in this paper. For ease of reference, we list the EIS datasets together with some pertinent information in Table 1. The datasets are the same as used by Warren et al. (2012) to study the emission measure (EM) distributions in the hot AR core loops, so many details of the observations are also available in that paper.

EIS data are affected by a number of instrumental issues that need addressed prior to analysis. There is a dark current pedestal that should be removed, and there are warm, hot, and dusty pixels, and others that have been struck by cosmicrays. We removed these effects using the eis_prep routine in SolarSoftware (SSW, Freeland \& Handy 1998). The centroid positions of the spectral lines also show a periodic variation around the satellite orbit as a result of thermal variations in the instrument structure. We removed this effect using a neural network model that relates velocity shifts to instrument temperatures (Kamio et al. 2010).

We show example spectral images for two of the datasets we analyzed in Figures 1 and 2 We prepared these images by fitting single Gaussians to the observed spectra, except for Fe XII 195.119 $\AA$, where we used a double Gaussian fit to account for the density sensitive blend in the red wing at $195.18 \AA$ (Del Zanna \& Mason 2003). The figures also indicate the boxed regions that we used for analysis of the hot core loops, and the fits shown in the lower panels are example fits to the spectra averaged within these boxes.

Regarding our analysis strategy, it is important to note that we work with uncalibrated counts (photons detected on the $\mathrm{CCD}$ ) rather than absolute physical units derived from applying the radiometric calibration. Although the EIS effective areas are a slowly varying function of wavelength (Lang et al. 2006), convolving them with the observed spectrum potentially introduces another uncertainty into the analysis; especially since the EIS sensitivity has evolved over time (Mariska 2013), and there are different methods of characterizing that evolution (Del Zanna 2013; Warren et al. 2014). Furthermore, the errors on the calibrated intensities also depend on wavelength, and have an important bearing on the spectral fits. This can be significant, depending on the treatment of the bad pixels. When we work in counts, the error is simply the square root of the intensity, with no other complicating factors.

We demonstrate the effect of the absolute calibration quantitatively in Figure 3, where we show scatter plot comparisons of the calibrated and uncalibrated line widths and nonthermal velocities for the AR shown in Figure 1 Although the calibrated and uncalibrated line widths are highly correlated, some scatter is evident, and there is a tendency for the calibrated widths to be larger, though we stress that this may depend on the dataset. In our example, the calibrated widths are larger overall in about $90 \%$ of the pixels. When we eliminate the weakest pixels - we use a threshold of $10 \%$ of the maximum intensity in Figure 1 - the differences are $\sim 10-20 \%$. These differences, however, amplify when propagated through to the non-thermal velocity computations. The scatter becomes larger, and factor of 4.5 differences are possible. In most cases, the differences are less than this ex- treme, but a 10-20\% change in the average width for the Fe XII $192.394 \AA$ line in this dataset produces a $15-26 \mathrm{~km} \mathrm{~s}^{-1}$ change in non-thermal velocity. Furthermore, cases where the thermal broadening is sufficient to explain the uncalibrated line width can produce erroneously large values. These findings imply that previous measurements of line widths and non-thermal velocities using calibrated EIS spectra should be treated with caution. Avoiding these additional uncertainties, together with the fact that for our study of line widths no absolute calibration is necessary, is the reason we analyze the raw data prior to applying any absolute calibration.

Note that the non-thermal velocities shown in Figure 3 are calculated from

$$
\delta \lambda=\frac{\lambda_{0}}{c} \sqrt{4 \ln 2\left(\frac{2 k_{B} T_{i}}{m}+\xi^{2}\right)+\sigma_{I}^{2}}
$$

where $\delta \lambda$ is the observed line width, $\lambda_{0}$ is the line centroid, $k_{B}$ is Boltzmann's constant, $T_{i}$ is the ion temperature, $m$ is the mass, $\xi$ is the non-thermal velocity, and $\sigma_{I}$ is the instrumental width.

The instrumental width is a key parameter in the analysis. It was measured in the laboratory prior to launch (Korendyke et al. 2006) and the full width at half maximum (FWHM) was found to be $0.047 \AA$ for the SW channel using the Mg III $187 \AA$ line and $0.055-0.057 \AA$ for the LW channel using He II $256 \AA$ and Ne III $267 \AA$. Brown et al. (2008) examined solar spectra acquired by Hinode in orbit post-launch and found evidence that the SW instrumental width is broader $(0.054 \AA)$ than measured on the ground in the Rutherford Appleton Laboratory. Hara et al. (2011) investigated the EIS instrumental width in detail by comparison of observed Fe XIV lines with ground based observations of the Fe XIV $5303 \AA$ green line obtained at the Norikura Solar Observatory for an AR on the west limb in 2007, July 18. They do not report values for the SW channel, but their Figure 17 implies an average value of $\sim 0.062 \AA$ for the LW channel. They find only a gradual variation with wavelength, but more importantly, a significant increase from $\sim 0.050 \AA$ to $\sim 0.068 \AA$ with Y-position on the CCD. This effect is also discussed in detail by Young (2011), who modeled the Yvariation of the instrumental width derived from the Fe XII $193.509 \AA$ line in several off-limb quiet Sun datasets obtained in 2009. Young (2011) provided his correction as an IDL routine (eis_slit_width) in SSW, and we use this program to correct for the $\bar{Y}$-variation in this paper by constructing an array of pixel values using the Y-start position of the observations, which is available in the EIS fits file header. In cases where we average over a small area, we also average the Ycorrection values.

The instrumental profile itself was found to be well represented by a Gaussian function in the laboratory (Korendyke et al. 2006) and we have independently verified this by re-examining the data for Ne III $267 \AA$. Deviations from a Gaussian profile may therefore be important signatures of the heating process. For example, numerical simulations of nanoflare heating predict some combination of Doppler shifted or highly distorted line profiles in the early phase, and subtle asymmetries later in the evolution (Patsourakos \& Klimchuk 2006). Although EIS line profiles have been found to often show important asymmetries (Hara et al. 2008a), that does not seem to be the case for the fits to the profiles for the hot core loops, as can be seen in 

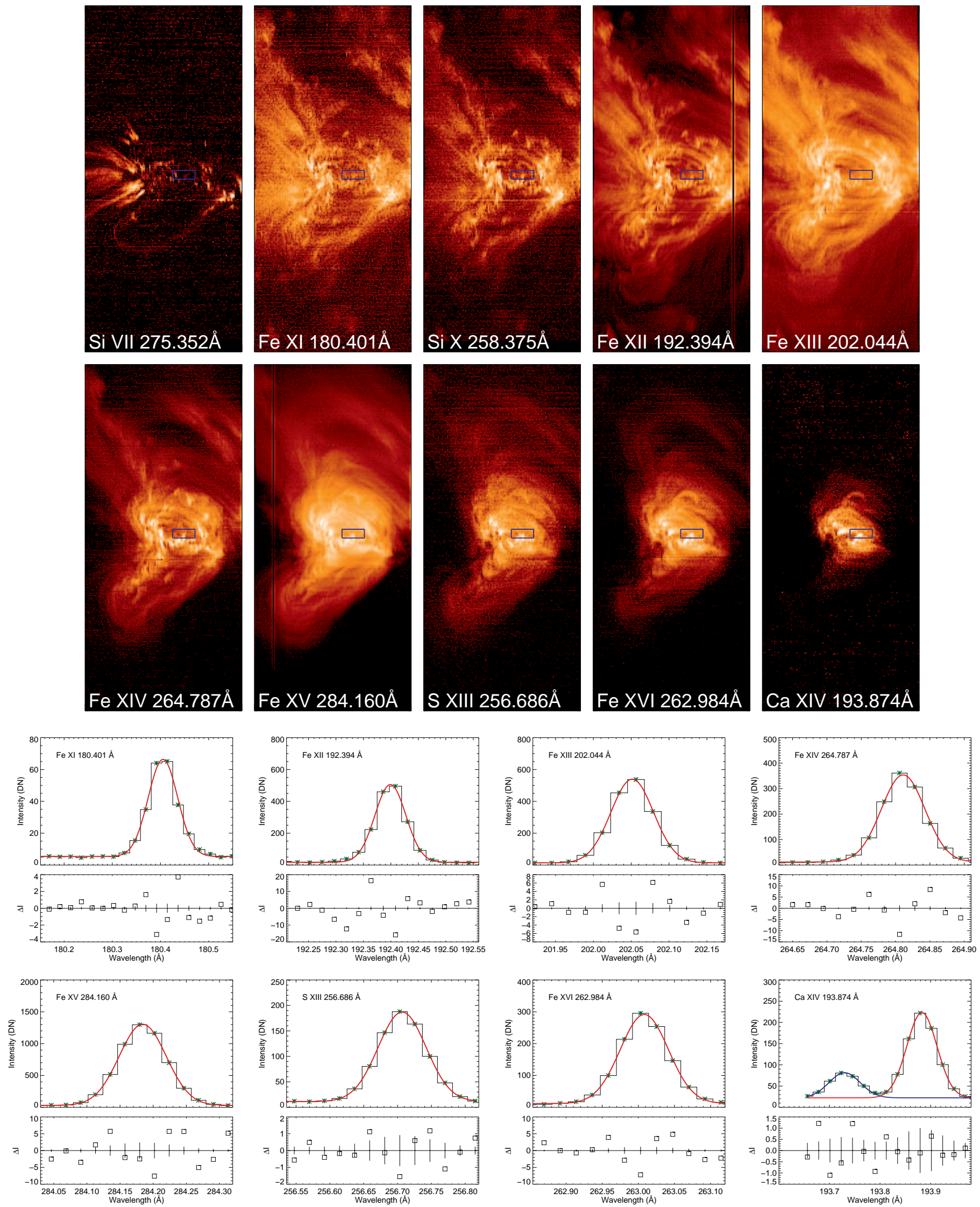

FIG. 2.- Example spectral images of AR 1190 observed on 2011, April 11. We measured the line widths in the high temperature core loops for the region indicated by the blue box. Example fits for several of the spectral lines are shown in the lower panels. 

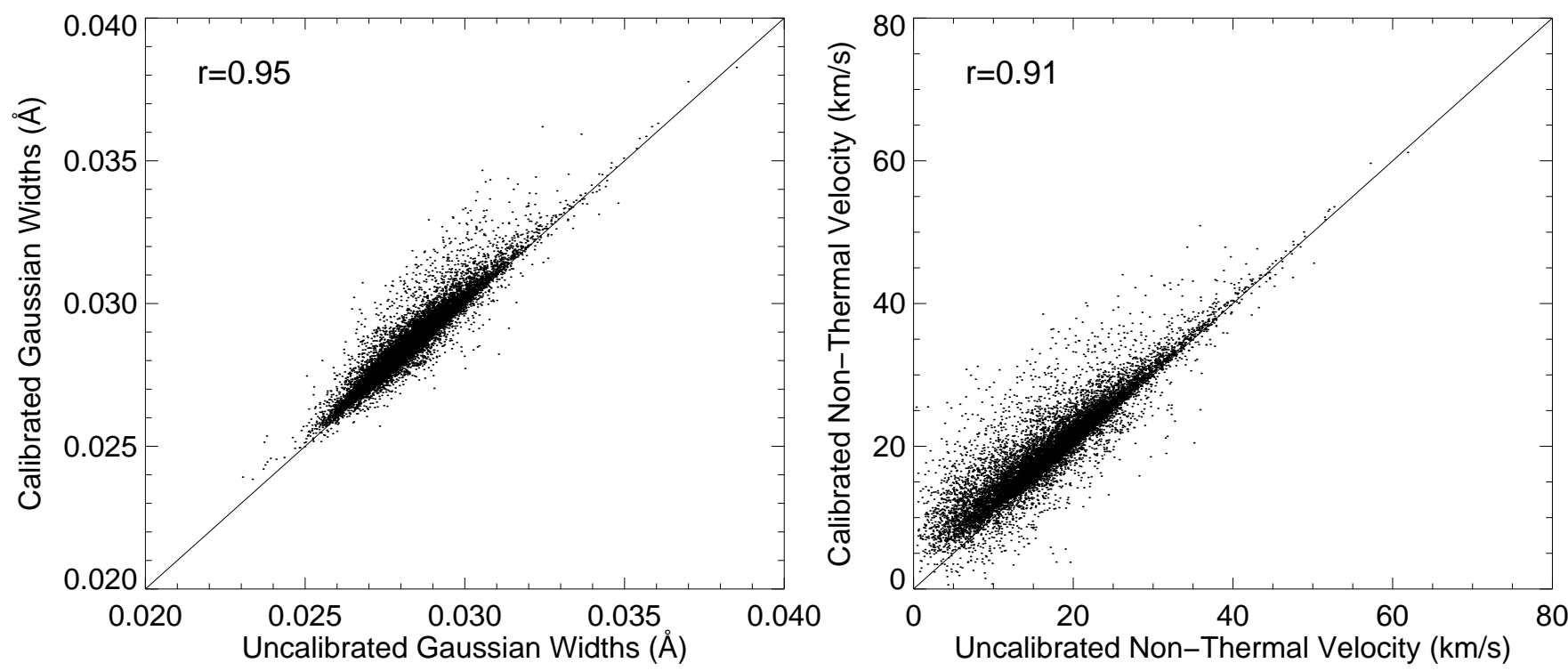

FIG. 3.- A comparison of line widths derived from Gaussian fits to calibrated and uncalibrated line profiles. Gaussian widths are shown on the left and the resulting non-thermal velocities are shown on the right. These calculations are for Fe XII 192.394 $\AA$ from the 2011, April 19 dataset in Figure 1 Only pixels that are brighter than $10 \%$ of the maximum intensity pixel are shown.
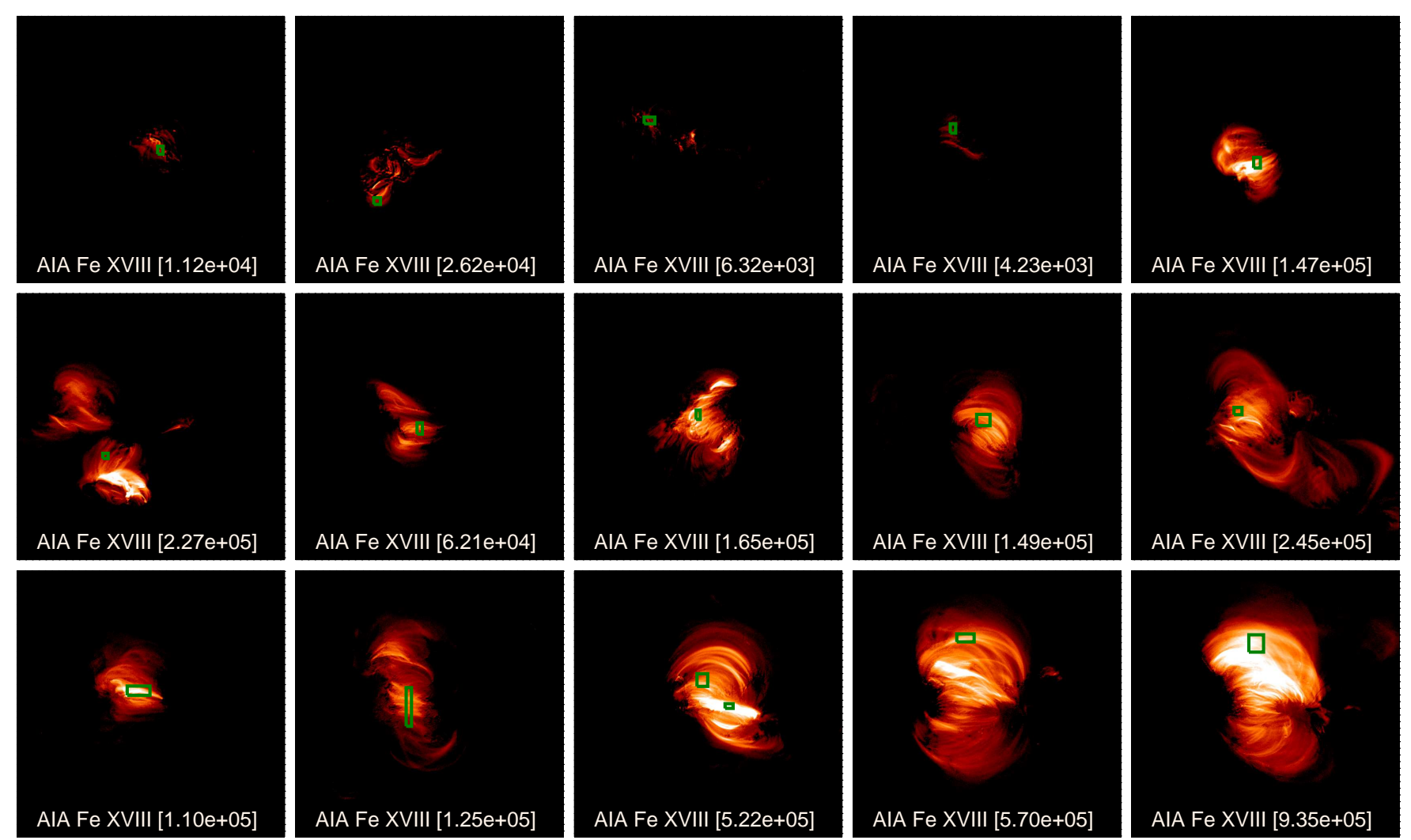

FIG. 4.- Fe XVIII $93.92 \AA$ images of the hot core loops analyzed. These were extracted from the AIA $94 \AA$ images. The green boxes show the locations where the EIS measurements are made. The number shown is the total intensity in the field-of-view.

Figures 1 and 2] Nevertheless, since we are attempting to measure the line widths as accurately as possible, we experimented with fits using a Voigt function with a fixed damp- ing factor based on a free-fit Gaussian function combined with a Lorentzian constructed from the measured instrumental width. These fits were quite close to a Gaussian, but showed 

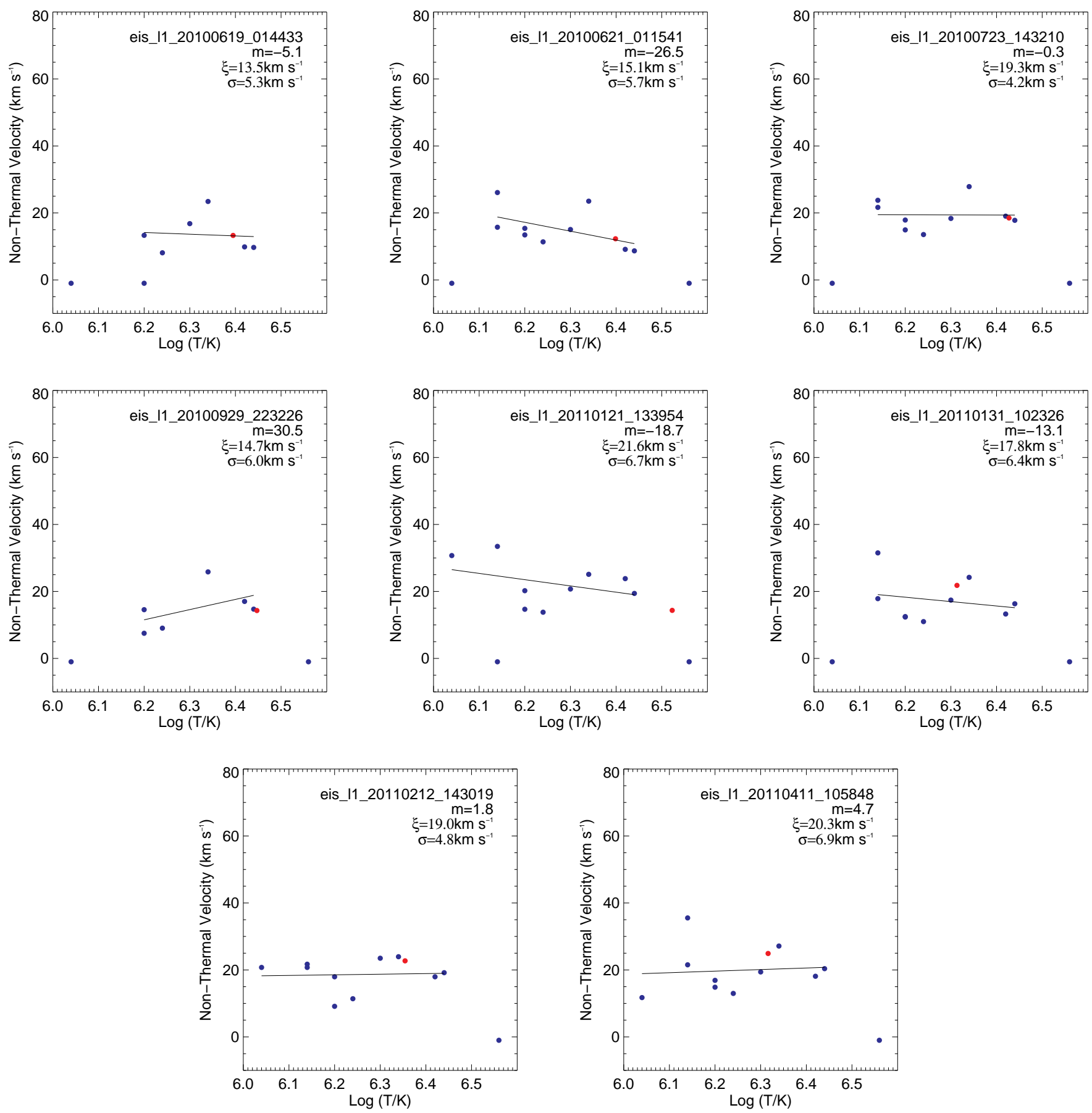

FIG. 5.- Plots of non-thermal velocity as a function of temperature for 8 of the active region cores. The datasets, gradient of the overlaid linear fit (m), mean non-thermal velocity $(\xi)$, and standard deviation $(\sigma)$ are indicated in the legend. The blue dots are the non-thermal velocities for each spectral line with an entry in Table 2 for the corresponding dataset (cross-referenced in the Table and plot legend). The red dot is the non-thermal velocity calculated using the method of Imada et al. (2009) (see text). Data from all lines are included here.

greater dispersion, indicating that we would gain no advantage from moving to a more complex fitting model. Furthermore, we closely examined the details of the Gaussian fits in Figures 1 and 2, and we found that the total residual intensity within $\pm 100 \mathrm{~km} \mathrm{~s}^{-1}$ of the line centroids was always less than $5 \%$ of the total intensity within the same wavelength range, indicating that the deviation from a Gaussian is small. This deviation also appears to decrease with temperature, making a
Gaussian function even more appropriate for the highest temperature lines which are of the most interest for this study. For all of these reasons, and also for ease of comparison with the literature results, we use only a simple Gaussian function for the fits in this paper. Note that we also use relatively narrow spectral windows for our fits. This is to avoid interference from background in the wings of the profiles (see the discussion related to Ca XIV 193.874 $\AA$ below). 

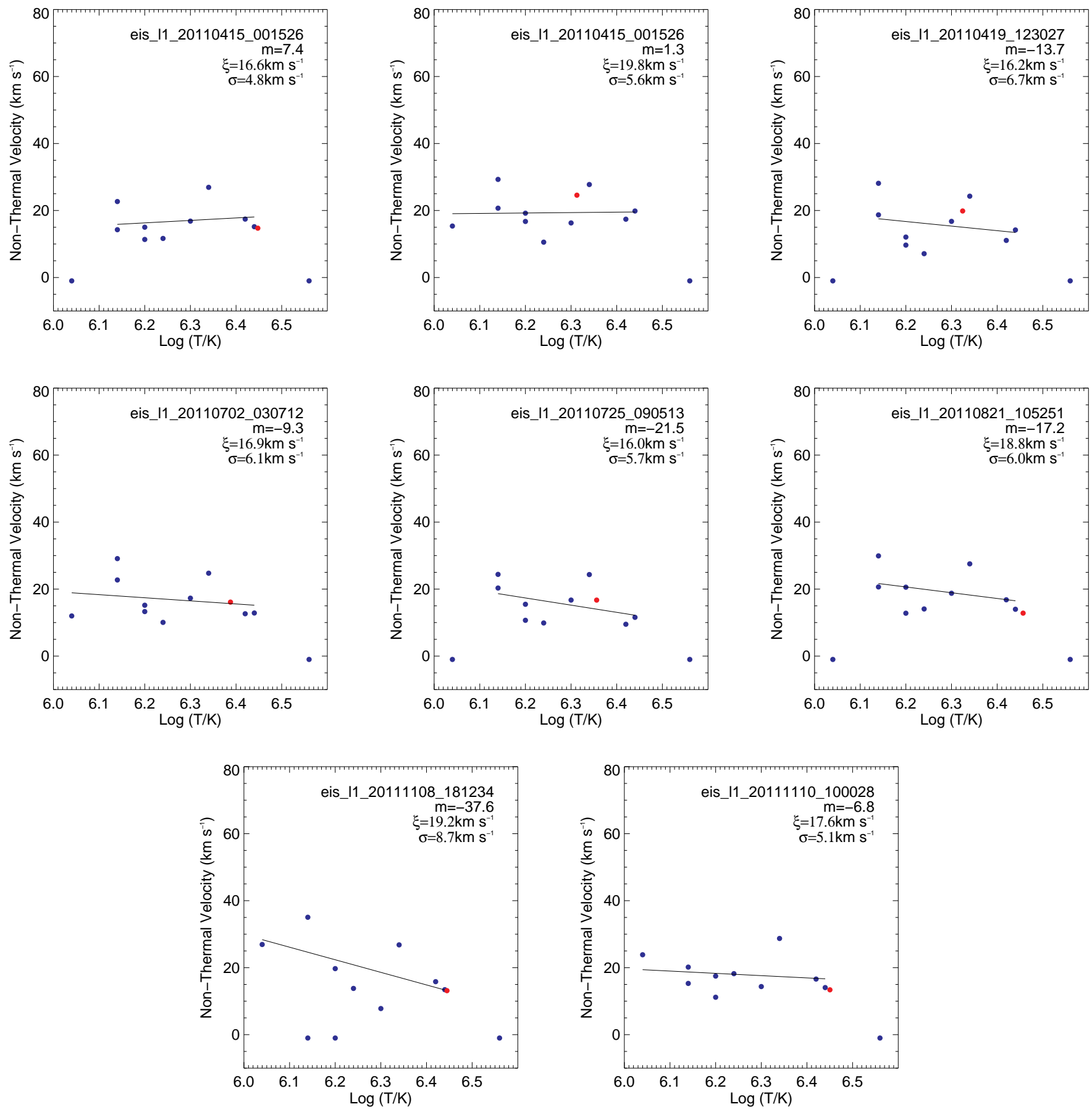

FIG. 6. - Plots of non-thermal velocity as a function of temperature for 8 of the active region cores. The datasets, gradient of the overlaid linear fit (m), mean non-thermal velocity $(\xi)$, and standard deviation $(\sigma)$ are indicated in the legend. The blue dots are the non-thermal velocities for each spectral line with an entry in Table 3 for the corresponding dataset (cross-referenced in the Table and plot legend). The red dot is the non-thermal velocity calculated using the method of Imada et al. (2009) (see text). Data from all lines are included here.

Ideally we would use only clean, unblended lines to obtain the best line width measurements, but since we are interested in any trend as a function of temperature, we have to compromise in some cases. Furthermore, most EIS datasets contain a limited number of spectral lines in order to conserve telemetry, so in some cases we have had to choose additional lines that we otherwise would not use. The lines we selected are given in Tables 2 and 3, and some more detailed comments on the reasons for selection are appropriate here.

First, Fe X 184.526 A, Fe XI 180.401 A, Fe XII 192.394 A, Fe XIII 202.044 $\AA$, Fe XIV 264.787 , Fe XVI 262.984 and Ca XIV $193.874 \AA$ are all clean, unblended and are either the strongest line for their ionization stage or have sufficient signal to produce good spectral fits. They cover the temperature range from $1.1-3.6 \mathrm{MK}$. Si X $258.275 \AA$ is also unblended and we include it in order to compare the Si X and 


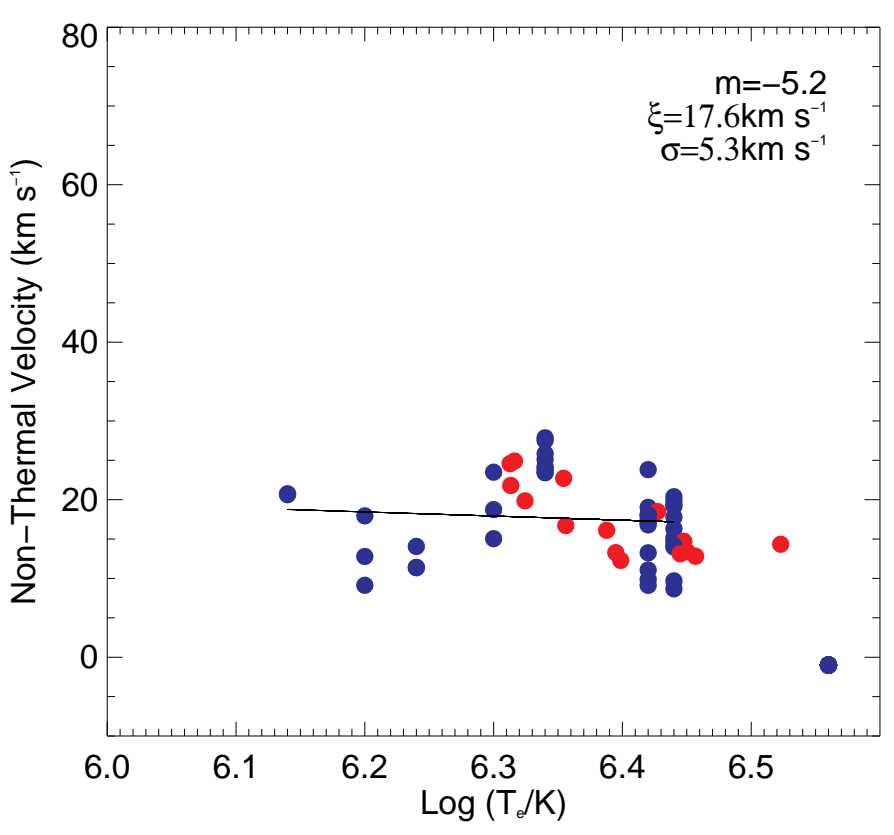

FIG. 7.- Non-thermal velocities as a function of line formation temperature for all 16 of the active region cores. Only lines with emission that is correlated with the emission of Ca XIV $193.874 \AA$ are included (see text). The quantities in the legend are the same as for Figures 5 and 6 except that the non-thermal velocities calculated using the Imada et al. (2009) method are only shown for comparison and are not included in the average or standard deviation. These values are discussed in relation to Figure 8

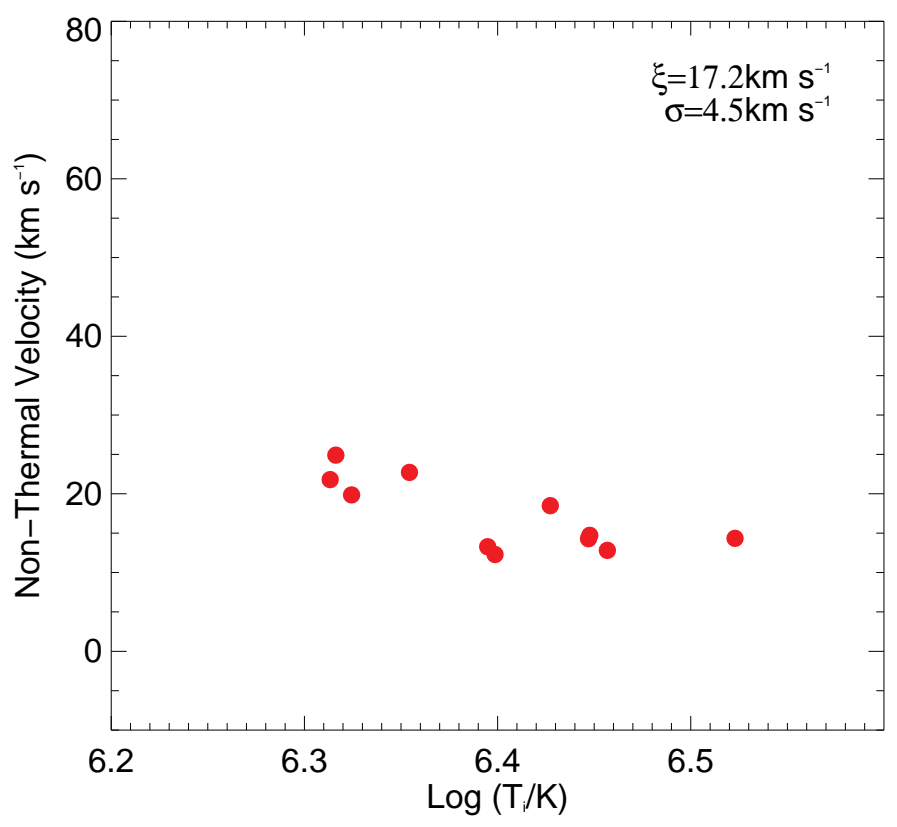

FIG. 8.- Non-thermal velocities calculated using the method of Imada et al. (2009) for all 16 of the active region cores. They are plotted as a function of the calculated ion temperature. Unlike Figures 5 6 and 7 the trend is not a function of temperature within an AR core. Rather it is a trend between different AR cores. To avoid confusion, we have not drawn a linear fit to these data.

Fe XII widths. Second, since not all of our datasets include the Fe XIV $264.787 \AA$ line, we added Fe XIV $274.203 \AA$ as a backup choice. We also include Fe XV 284.160 ̊. This is a strong line and fills the temperature gap between Fe XIV and Fe XVI, which is otherwise quite large. We also include S XIII $256.686 \AA$ for comparison with Fe XVI 262.984 $\mathrm{A}$. These last three lines are weakly blended, Fe XIV $274.203 \AA$ with Si VII $274.175 \AA$ and Fe XV 284.160 $\AA$ with Al IX 284.015 $\AA$ and S XIII 256.686 $\AA$ with Ni XVI $256.62 \AA$, but we expect any systematic deviation in line width to become apparent in comparison with the other lines.

In addition to computing non-thermal velocities using Equation 1, and as an independent check, we also calculate them using a different method outlined by Imada et al. (2009) (also see Seely et al. 1997). Imada et al. (2009) used lines from ions of different masses, and, under the assumption that the non-thermal velocity and ion temperature are the same, derived expressions from which they could be computed.

$$
\xi^{2}=\frac{m_{2} W_{2}^{2}-m_{1} W_{1}^{2}}{4 \log 2\left(m_{2}-m_{1}\right)}
$$

and

$$
T_{i o n}=\frac{W_{1}^{2}-W_{2}^{2}}{8 k_{B} \log 2} \frac{m_{1} m_{2}}{m_{2}-m_{1}}
$$

where $W=\delta \lambda^{2}-\sigma_{I}^{2}$, and 1 and 2 indicate the ion species. Imada et al. (2009) used S XIII 256.686 $\AA$ and Fe XVI $262.984 \AA$ for their study, and we use the same line pair here.

We analyzed 16 high temperature core loop arcades from the 15 datasets listed in table 1 . Figure 4 shows Fe XVIII 93.92 $\AA$ images of the ARs that were extracted from AIA $94 \AA$ filtergrams using the technique described by Warren et al. (2012). The green boxes indicate the regions where the measurements were made. These regions were carefully selected to isolate the loops that are bright in Fe XVIII $93.92 \AA$, with minimal contamination from footpoint (moss) emission below. The AIA $193 \AA$ filtergrams were then coaligned with the EIS Fe XII 195.119 $\AA$ raster images, and the relevant areas extracted for all of the lines of interest. More details of the selection and coalignment methods are given in Warren et al. (2012).

The results of our measurements are displayed in Figures 5. 6 and the values are noted explicitly in Tables 2, 3. Figures 5 and 6 show the measured non-thermal velocities plotted as a function of temperature for the 16 core loop arcades. The non-thermal velocities are all less than $35 \mathrm{~km} \mathrm{~s}^{-1}$ with mean values in the range $13.5-21.6 \mathrm{~km} \mathrm{~s}^{-1}$ with a standard deviation of $4.2-8.7 \mathrm{~km} \mathrm{~s}^{-1}$. Linear fits to the non-zero measurements are overlaid to draw the eye to any trend in the data, but taking into consideration the scatter in the measurements, there is no statistically significant trend.

We present the complete results in the figures and tables so that they are on record, but it is not necessarily the case that the emission at all temperatures comes from the hot core loops. If that were the case we would expect these loops to have broad EMs whereas Warren et al. (2012) found strongly peaked EM distributions with a sharp fall below and above the peak temperature in most cases. In some of the weaker ARs a shallower temperature distribution was observed, and this highlights the need to adopt some method of quantitatively determining if the emission comes from the hot core loops or not. In our previous EM studies of warm coronal loops (Warren et al. 2008b; Brooks et al. 2012, 2013) we have adopted the criterion that the cross-field intensity profile must 
TABLE 2

LiNE FWHMS AND NON-THERMAL VELOCITIES

\begin{tabular}{|c|c|c|c|c|c|c|c|c|}
\hline \multirow[b]{2}{*}{ Line ID } & \multicolumn{6}{|c|}{ Active Region Observation Date } & \multirow[b]{2}{*}{20110212} & \multirow[b]{2}{*}{20110411} \\
\hline & 20100619 & 20100621 & 20100723 & 20100929 & 20110121 & 20110131 & & \\
\hline Fe X 184.536 & $0.069[0.00]$ & $0.061[0.00]$ & $0.062[0.00]$ & $0.062[0.00]$ & 0.069 [30.7] & $0.062[0.00]$ & 0.065 [20.7] & 0.063 [11.7] \\
\hline Fe XI 180.401 & - & $0.068[26.0]$ & $0.067[23.7]$ & - & $0.071[33.4]$ & $0.070[31.5]$ & 0.066 [21.7] & $0.072[35.5]$ \\
\hline Fe XII 192.394 & $0.073[13.3]$ & $0.066[13.4]$ & $0.067[17.8]$ & $0.066[14.5]$ & $0.067[20.2]$ & $0.065[12.4]$ & 0.066 [17.9] & $0.066[16.8]$ \\
\hline Fe XII 195.119 & $0.071[0.00]$ & $0.066[15.3]$ & 0.066 [14.9] & $0.065[7.51]$ & $0.065[14.6]$ & $0.065[12.3]$ & $0.064[9.13]$ & $0.066[14.8]$ \\
\hline Fe XIII 202.044 & $0.073[8.10]$ & $0.066[11.3]$ & $0.067[13.5]$ & $0.066[9.03]$ & $0.066[13.8]$ & $0.066[10.9]$ & $0.065[11.4]$ & 0.066 [12.9] \\
\hline Fe XIV 264.787 & - & $0.073[15.0]$ & $0.075[18.3]$ & - & $0.075[20.7]$ & $0.074[17.4]$ & $0.077[23.5]$ & $0.075[19.4]$ \\
\hline Fe XIV 274.203 & $0.081[16.8]$ & - & - & - & - & - & - & - \\
\hline Fe XV 284.160 & $0.087[23.4]$ & 0.081 [23.5] & 0.084 [27.8] & $0.083[25.8]$ & $0.081[25.1]$ & 0.081 [24.2] & 0.080 [23.9] & $0.084[27.1]$ \\
\hline Fe XVI 262.984 & $0.081[9.70]$ & $0.074[8.68]$ & $0.077[17.7]$ & $0.076[14.7]$ & $0.077[19.4]$ & $0.077[16.3]$ & $0.077[19.1]$ & $0.078[20.3]$ \\
\hline Si X 258.375 & - & $0.076[15.7]$ & $0.079[21.6]$ & - & $0.067[0.00]$ & $0.077[17.8]$ & $0.077[20.7]$ & $0.078[21.5]$ \\
\hline S XIII 256.686 & $0.087[9.86]$ & $0.081[9.13]$ & $0.084[19.0]$ & $0.083[17.0]$ & $0.086[23.8]$ & $0.082[13.2]$ & 0.083 [17.9] & $0.083[18.1]$ \\
\hline Ca XIV 193.874 & - & $0.066[0.00]$ & $0.072[0.00]$ & $0.069[0.00]$ & $0.069[0.00]$ & $0.072[0.00]$ & $0.065[0.00]$ & $0.070[0.00]$ \\
\hline$\xi$ & 13.2 & 12.2 & 18.4 & 14.3 & 14.3 & 21.8 & 22.7 & 24.9 \\
\hline
\end{tabular}

The FWHMs are in units of $\AA$ and the non-thermal velocities are in $\mathrm{km} \mathrm{s}^{-1} . \xi$ is the non-thermal velocity calculated using the Imada et al. (2009) method.

TABLE 3

LINE FWHMS AND NON-THERMAL VELOCITIES

\begin{tabular}{|c|c|c|c|c|c|c|c|c|}
\hline \multirow[b]{2}{*}{ Line ID } & \multicolumn{6}{|c|}{ Active Region Observation Date } & \multirow[b]{2}{*}{20111108} & \multirow[b]{2}{*}{20111110} \\
\hline & 20110415 & 20110415 & 20110419 & 20110702 & 20110725 & 20110821 & & \\
\hline Fe X 184.536 & $0.062[0.00]$ & $0.064[15.3]$ & $0.059[0.00]$ & $0.063[11.9]$ & $0.063[0.00]$ & $0.061[0.00]$ & $0.071[26.9]$ & $0.069[23.8]$ \\
\hline Fe XI 180.401 & $0.067[22.6]$ & $0.069[29.2]$ & $0.069[28.1]$ & $0.069[29.1]$ & $0.068[24.3]$ & $0.069[29.9]$ & $0.075[35.0]$ & $0.068[20.1]$ \\
\hline Fe XII 192.394 & $0.066[15.0]$ & $0.067[19.2]$ & $0.065[12.0]$ & $0.065[15.1]$ & $0.067[15.4]$ & $0.067[20.5]$ & $0.070[19.7]$ & $0.069[17.4]$ \\
\hline Fe XII 195.119 & $0.066[11.3]$ & $0.066[16.7]$ & $0.065[9.67]$ & $0.065[13.2]$ & $0.066[10.6]$ & $0.065[12.8]$ & $0.066[0.00]$ & $0.067[11.1]$ \\
\hline Fe XIII 202.044 & $0.067[11.6]$ & $0.066[10.5]$ & $0.066[7.11]$ & $0.065[10.0]$ & $0.067[9.88]$ & $0.066[14.0]$ & $0.069[13.8]$ & $0.070[18.2]$ \\
\hline Fe XIV 264.787 & $0.074[16.8]$ & $0.073[16.2]$ & $0.074[16.7]$ & $0.074[17.2]$ & $0.075[16.7]$ & $0.074[18.7]$ & $0.073[7.80]$ & $0.075[14.3]$ \\
\hline Fe XV 284.160 & 0.084 [26.9] & $0.084[27.7]$ & $0.082[24.2]$ & $0.081[24.7]$ & $0.082[24.3]$ & $0.084[27.5]$ & 0.086 [26.8] & 0.087 [28.7] \\
\hline Fe XVI 262.984 & $0.076[15.1]$ & 0.078 [19.8] & $0.076[14.1]$ & $0.075[12.8]$ & $0.076[11.5]$ & $0.075[13.9]$ & $0.078[13.4]$ & $0.078[14.0]$ \\
\hline Si X 258.375 & $0.076[14.2]$ & $0.078[20.6]$ & $0.077[18.7]$ & $0.079[22.7]$ & $0.079[20.3]$ & $0.078[20.6]$ & $0.070[0.00]$ & $0.078[15.3]$ \\
\hline S XIII 256.686 & $0.084[17.4]$ & $0.083[17.4]$ & $0.081[11.0]$ & $0.081[12.6]$ & $0.081[9.50]$ & $0.083[16.8]$ & $0.085[15.8]$ & 0.085 [16.6] \\
\hline Ca XIV 193.874 & $0.070[0.00]$ & $0.070[0.00]$ & $0.069[0.00]$ & $0.067[0.00]$ & $0.064[0.00]$ & $0.069[0.00]$ & $0.071[0.00]$ & $0.072[0.00]$ \\
\hline$\xi$ & 14.7 & 24.5 & 19.8 & 16.1 & 16.7 & 12.8 & 13.1 & 13.3 \\
\hline
\end{tabular}

be highly correlated with that of the spectral line with which they were identified (usually Fe XII 195.119 ̊). Here we impose the condition that the emission within the boxed region for each spectral line must be spatially correlated $(r>0.6)$ with that of the highest temperature line (Ca XIV 193.874 $\AA$ ). The measurements satisfying this criterion for all the AR loops studied are shown in Figure 7 . Note that no lines formed at temperatures below $1.4 \mathrm{MK}$ pass this test.

Returning to the measurements, the mean value for all the hot loops is $17.6 \mathrm{~km} \mathrm{~s}^{-1}$ with a standard deviation of $5.3 \mathrm{~km}$ $\mathrm{s}^{-1}$, and as with the individual plots (Figures 5, 6) the two methods of non-thermal velocity calculation appear to give fairly consistent results.

Note that the highest temperature Ca XIV 193.874 $\AA$ measurements are always zero. This is inconsistent with the results for the other highest temperature line, Fe XVI 262.984 $\AA$, and also with previous ground based measurements of $\mathrm{Ca} X \mathrm{XV}$ $5694 \AA$ obtained with the Norikura solar observatory coronagraph by Hara \& Ichimoto (1999). The Norikura coronagraph has superior spectral resolving power to EIS and obtained values of $\sim 16-26 \mathrm{~km} \mathrm{~s}^{-1}$ for a limb AR, which would be fairly consistent with our other results. Something is therefore missing in our understanding of the EIS Ca XIV 193.874 $\AA$ line width measurements.

One possibility is that the Ca XIV $193.874 \AA$ measurement can be attributed to an unusual deviation of the instrumen- tal width. It lies very close to the Fe XII $192.394 \AA$ line which shows similar values for the measured line width within 7\%; see Tables 2 and 3 — and the Ca XIV $193.874 \AA$ non-thermal velocities could be brought into agreement with those of Fe XII $192.394 \AA$ if the instrumental width drops to $0.047 \AA$ at $193.874 \AA$. This would actually be in agreement with the SW laboratory measurement of the instrumental width, but in disagreement with most on orbit measurements. It is difficult to understand how the laboratory calibration could be maintained at one wavelength while degrading by $\sim 40 \%$ within $1.5 \AA$, however, so we consider this explanation unlikely.

Another possibility is that Ca XIV $193.874 \AA$ is formed at a much lower temperature than suggested by the ionization equilibrium calculations. The contribution function peaks at $3.6 \mathrm{MK}$, but if the line were actually formed below $1 \mathrm{MK}$ the non-thermal velocity measurements would be non-zero. We also consider this explanation unlikely since the EM distributions for all of these regions actually peak close to the equilibrium formation temperature of Ca XIV $193.874 \AA$ (Warren et al. 2012).

Another explanation for this observation is that the complex background in this region of the spectrum adversely affects the line profile fits. Since the background is high, sloped, and affected by several weaker lines, there is a possibility that the full height of the line profile is not exposed, and therefore 
that the width measurement is made slightly higher than the half maximum location. This issue can actually resolve the discrepancy between the Ca XIV $193.874 \AA$ non-thermal velocity measurements and the others, bringing the non-thermal velocities up to values of $19-33 \mathrm{~km} \mathrm{~s}^{-1}$. We have also independently verified that we can measure non-zero non-thermal velocities in the Ca XIV $193.874 \AA$ line during an X1.8 class solar flare when the line is more prominently exposed above the background. Since a definitive proof that this is the correct explanation requires higher spectral resolution observations of this line, however, we discuss it only in the Appendix.

Figure 8 shows the non-thermal velocity results calculated using Equation 2 and plotted against the ion formation temperature calculated using Equation 3 for the AR core loops where the emission from the diagnostic S XIII $256.686 \AA$ and Fe XVI $262.984 \AA$ lines is correlated with Ca XIV $193.874 \AA$. The average value in this case is $17.2 \mathrm{~km} \mathrm{~s}^{-1}$ with a standard deviation of $4.5 \mathrm{~km} \mathrm{~s}^{-1}$, which is consistent with the previous results shown. The ion temperatures fall in the range $2-3 \mathrm{MK}$, which are slightly lower than the temperature of the peak of the EM calculated by Warren et al. (2012), but not low enough to solve the Ca XIV $193.874 \AA$ non-thermal velocity discrepancy. The plot also shows a decreasing non-thermal velocity with increasing ion formation temperature of each AR. Note that this is a trend between ARs not within any single one.

Warren et al. (2012) provide other parameters for this sample of AR core loops in their Table 1 such as the total unsigned magnetic flux of the AR, the total AIA Fe XVIII intensity, and the gradient of the EM slope below and above the peak. We have verified that there is no correlation between any of these parameters and our measured non-thermal velocities.

Finally, we also investigated any relationship between the non-thermal velocities measured in Fe XVI 262.984 $\AA$ and the loop lengths, by attempting to trace the loops from the foot-point to the apex in the Fe XVIII $93.92 \AA$ images of Figure 4 The results are not always directly comparable: in most cases the tracing was satisfactory, but sometimes only part of the loop length was visible, or the boxed region where the non-thermal velocities were measured caught parts of other hot loops in the AR core; in which case we measured the length of another loop in the AR core arcade. While there were potentially interesting trends in some subsets of the data, in general we found the correlation between loop length and non-thermal velocity to be poor.

\section{SUMMARY AND DISCUSSION}

We have carried out a survey of non-thermal line widths in the high temperature (1.1-3.6MK) loops in the cores of 15 non-flaring ARs spanning a wide range of solar conditions. We compute the non-thermal velocities considering the instrumental and thermal broadening of the spectral lines in the usual way, and we also utilize the method outlined by Imada et al. (2009). The results from the two methods are broadly in agreement, and we find non-thermal velocities of $\sim 17 \mathrm{~km} \mathrm{~s}^{-1}$ on average. We also find no significant trend with temperature, or with any other property of the ARs such as total magnetic flux, or the slope of the loop EM distributions. We do, however, detect a tendency for AR loops with the highest ion temperatures to have smaller non-thermal velocities.

We note that stellar observers have concluded that nonthermal velocities below $\sim 20 \mathrm{~km} \mathrm{~s}^{-1}$ indicate that the spectral lines are basically thermally broadened (Linsky et al.
1998). While that is not strictly the case here, the modest values we find, and the lack of a temperature trend, are a challenge for current coronal heating models to explain. These are two of the key measurements that in combination can be used not just for discriminating between different coronal heating models, but also for distinguishing between the physical processes within the same model. For example, in the nanoflare model, reconnection jets are expected to exist while the plasma is still too hot to have cooled sufficiently for emission to appear at lower temperatures. So a comparison with the non-thermal velocity dependence on temperature is not appropriate. Conversely, emission at lower temperatures is dominated by the later phase after the nanoflare has ended and such a comparison becomes possible. We find that our measured non-thermal velocities are much smaller than predicted from either the high temperature reconnection jets in the nanoflare-heated corona model, or shock heating associated with Alfvén waves, both of which suggest velocities on the order of hundreds of $\mathrm{km} \mathrm{s}^{-1}$ (Cargill 1996; Antolin et al. 2008). Models of chromospheric evaporation in response to coronal nanoflares are closer to the observations around $1 \mathrm{MK}$, but predict that non-thermal velocities should increase with temperature. This is because the emission at cooler temperatures is low while chromospheric evaporation is occurring and is dominated by the later slow draining phase, whereas the emission at higher temperatures occurs earlier in the plasma evolution and is produced not just by draining but also by evaporation. This trend, however, is not observed, and leads to a discrepancy of a factor of 2 around $4 \mathrm{MK}$ (Patsourakos \& Klimchuk 2006). Some models of Alfvén wave turbulence also produce values that are closer to the observations around 1.6 MK, and show only limited variations with temperature at the loop apex, but they show a large spread of predictions, and require the imposition of a random flow component parallel to the magnetic field (Asgari-Targhi et al. 2014).

Of course, further modeling with different physical conditions may alter the predicted characteristics of the spectra. For example, non-equilibrium ionization will alter the line intensities, and this effect was recently demonstrated to be significant in the case of flare-like reconnection jets (Imada et al. 2011). A change in the line intensity implies a potential alteration of the line width. It seems more intuitive to think that a more violent plasma will produce more dynamic spectral signatures, but it is possible that this is not the case, and it should be checked with numerical simulations. Temperature trends could also be affected.

For the nanoflare model it is possible that high temperature reconnection jets exist, but we are unable to observe them in AR core loops because the EM is too low (as discussed earlier). Non-equilibrium ionization could contribute to surpressing the emission too (Bradshaw \& Cargill 2006). In this picture, the non-thermal broadening of the spectral lines would appear as it does in real flares, with the hot AR core loops corresponding to post-flare loops observed in their cooling phase. In fact, EIS observations of flares do sometimes show substantial line broadening at high temperatures. Hara et al. (2008b) observed a long-duration event on the limb, and measured significant non-thermal velocities of $125 \mathrm{~km} \mathrm{~s}^{-1}$ at the top of cusp-shaped flare loops in the Ca XVII 192.858 $\AA$ line formed near 5.6 MK. Hara et al. (2008b) and Doschek et al. (2014), both noted that the non-thermal velocities at coronal temperatures in flares $(1.1-2.2 \mathrm{MK})$ tend to be smaller $(10$ 
$40 \mathrm{~km} \mathrm{~s}^{-1}$ ), which is consistent with the velocities diminishing as flare loops cool. Hara et al. (2008b) actually made the measurements in post-flare loops on the limb.

These results would appear to fit the idea that the heating of AR core loops is truly flare-like, but we only observe the non-thermal broadening that remains well after the energy release process is complete. In this sense, measurements of non-thermal broadening are not a good diagnostic of the heating phase. In fact there have also been studies showing tentative signals of cooling plasma in the hot cores of ARs (Viall \& Klimchuk 2012).

The picture for flares, however, is incomplete. Doschek et al. (2014), for example, only measured values of $20-60 \mathrm{~km} \mathrm{~s}^{-1}$ in Fe XXIV in their sample of $\mathrm{M}$ - and $\mathrm{X}$-class flares. Furthermore, there is a body of evidence that suggests that the hot loops in the cores of ARs are not cooling, but instead are maintained at high temperatures by continual high frequency heating (Antiochos et al. 2003; Brooks \& Warren 2009; Warren et al. 2010).

This work cannot draw a firm conclusion on the heating process, but we present a systematic observational study of non-thermal broadening in high temperature core loops that provides an important observational constraint for coronal heating models.

The authors would like to thank Peter Cargill for suggesting this project. This work was funded by the NASA Hinode program. Hinode is a Japanese mission developed and launched by ISAS/JAXA, with NAOJ as domestic partner and NASA and STFC (UK) as international partners. It is operated by these agencies in co-operation with ESA and NSC (Norway).

\section{APPENDIX}

\section{BACKGROUND INFLUENCE ON THE CA XIV 193.874 Å LINE PROFILE}

The spectral interval immediately surrounding the Ca XIV $193.874 \AA$ line is fairly crowded, with the following lines present according to Brown et al. (2008): Fe X 193.715 $\AA$, Fe VIII 193.967 $\AA$, a blend of Ni XVI 194.046 $\AA$ and Ar XI $194.104 \AA$, and Ar XIV 194.396 $\AA$. There are also unidentified lines at $194.227 \AA$ and $194.315 \AA$. The background level is also sloped and difficult to determine, and as discussed in the main paper, these effects may partially conceal the Ca XIV $193.874 \AA$ line profile and result in the width measurement at half-maximum intensity actually being taken slightly above the half maximum. This is illustrated in Figure 9, which shows a sample spectrum in this wavelength region. The blue and red fits to the data (black histogram) are made by selecting slightly different points as initial guesses for a linear background fit in order to test cases where the background is either well matched close to or far from the Ca XIV $193.874 \AA$ line profile. Note that the intensity scale is logarithmic in order to highlight the varying background levels, so the differences in these fits are small, and the width measurements for Ca XIV $193.874 \AA$ are not substantially affected.

The background level near Ar XIV 194.396 $\AA$, however, is $\sim 2$ times smaller than the background level on the long wavelength side of Ca XIV 193.874 $\AA$ and $\sim 3$ times smaller than on the short wavelength side. The variation of the background depending on where it is measured is illustrated by the horizontal green lines. The template fits for the results in the main part of the paper measure the background close to the line profile where it is relatively high, however, the actual measured width as a result of the more elaborate fit shown in Figure 9 is not significantly different, $0.031 \AA$ Gaussian width compared to $0.030 \AA$, and cannot resolve the discrepancy in non-thermal velocities. Conversely, the base of the Ca XIV $193.874 \AA$ line appears partly hidden by the sloping background and blending with the wings of the Fe X $193.715 \AA$ and Fe VIII $193.967 \AA$ lines.

Without higher spectral resolution observations we cannot confirm that the profile is affected, but we have performed a series of experiments to simulate what the effect would be. These are illustrated in Figure 10. The top left panel shows a simulated Ca XIV 193.874 $\AA$ line profile (black histogram) with Gaussian fits to randomly selected profiles with successively higher background levels in blue. The initial profile is constructed with a Gaussian area of $10000 \mathrm{DN}$ so that the intensity error is less than $1 \%$ of the total intensity. The Gaussian line width was chosen as $0.030 \AA$. This profile is then fit with a Gaussian function plus linear background and the background fit extracted. The background is then increased, re-convolved with the original Gaussian part, and a further Gaussian fit is made. This process is repeated until the Gaussian part of the function is completely swamped by the background.

We extracted the line width from the Gaussian fits to each simulated profile, and plotted them as a function of the profile peak intensity to average background ratio in the top right panel of Figure 10. We can see that the line width is a slowly decreasing function of the ratio, but decreases rapidly as the Gaussian part of the profile disappears. To examine the significance of the effect, we plotted the percentage change in the line width (compared to the starting value) as a function of the same profile peak intensity to average background ratio in the lower left panel of Figure 10. The width changes by less than $10 \%$ until the ratio is larger than $\sim 15$, but we know from Figure 3 in the main paper that line width changes of that order can result in significant changes in computed non-thermal velocities. So we have overlaid red dots on the figure at the locations of the actual measured profile peak intensity to average background ratio for the 16 AR core loops in our study, to determine how much each of our actual measurements would be affected. From the Figure it appears that our line width measurements could change by $9-25 \%$, which is significant.

The lower right panel of Figure 10 shows arrows indicating the change in computed non-thermal velocity for the sample of Ca XIV 193.874 $\AA$ measurements as a result of the percentage changes indicated by the lower left panel. Our experiments imply that when the background effect is taken into account, the revised Ca XIV 193.874 $\AA$ non-thermal velocities for the sample AR core loops fall in the range $19-33 \mathrm{~km} \mathrm{~s}^{-1}$ with an average value of $25 \mathrm{~km} \mathrm{~s}^{-1}$. This is broadly in agreement with the results for the other lines within the uncertainties. As noted in the main text, we have also verified that we can measure non-zero values for Ca XIV 193.874 $\AA$ in an X1.8 solar flare when the line is more prominent above the background. This supports the arguments made here. 


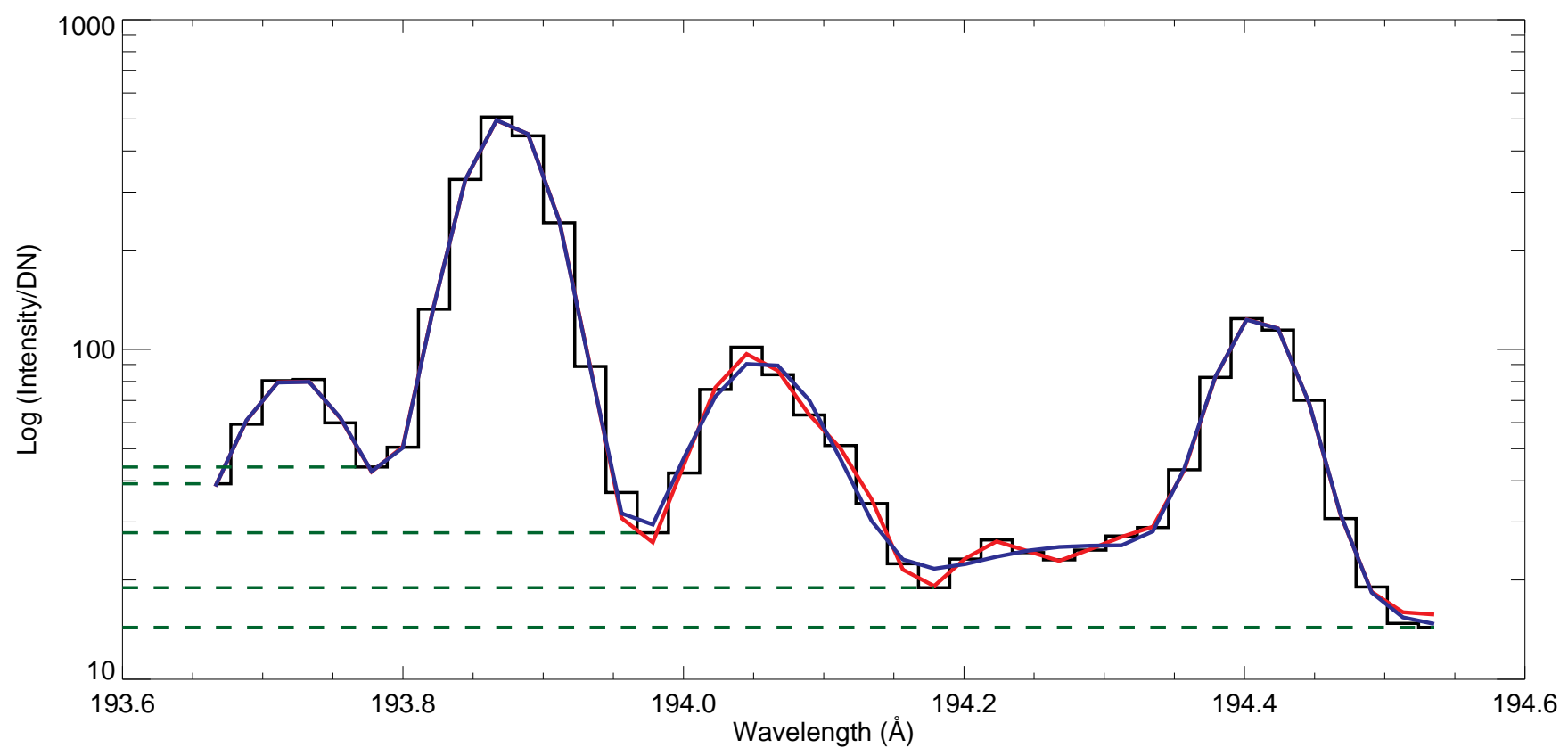

FIG. 9.- Sample spectrum close to Ca XIV 193.874 A (black histogram). The data are of the hot AR core loop observed on 2010, September 29. The blue and red lines show example fits using different locations for a linear fit to the background level. The horizontal dashed green lines indicate the variation in background level depending on the spectral pixel where it is measured. The intensity scale is logarithmic to highlight the variation in background. There are seven spectral lines within this wavelength range (see text).

\section{REFERENCES}

Acton, L. W., et al. 1981, ApJ, 244, L137

Antiochos, S. K., Karpen, J. T., DeLuca, E. E., Golub, L., \& Hamilton, P 2003, ApJ, 590, 547

Antolin, P., Shibata, K., Kudoh, T., Shiota, D., \& Brooks, D. 2008, ApJ, 688 669

Arregui, I. 2015, Philosophical Transactions of the Royal Society of London Series A, 373, 40261

Asgari-Targhi, M., van Ballegooijen, A. A., \& Imada, S. 2014, ApJ, 786, 28

Baker, D., van Driel-Gesztelyi, L., Mandrini, C. H., Démoulin, P., \& Murray, M. J. 2009, ApJ, 705, 926

Bradshaw, S. J., \& Cargill, P. J. 2006, A\&A, 458, 987

Brooks, D. H., Ugarte-Urra, I., \& Warren, H. P. 2015, Nature Communications, 6, 5947

Brooks, D. H., \& Warren, H. P. 2009, ApJ, 703, L10

Brooks, D. H., \& Warren, H. P. 2011, ApJ, 727, L13

Brooks, D. H., Warren, H. P., \& Ugarte-Urra, I. 2012, ApJ, 755, L33

Brooks, D. H., Warren, H. P., Ugarte-Urra, I., \& Winebarger, A. R. 2013, ApJ, 772, L19

Brown, C. M., Feldman, U., Seely, J. F., Korendyke, C. M., \& Hara, H. 2008, ApJS, 176, 511

Cargill, P. J. 1996, Sol. Phys., 167, 267

Cheng, C.-C., Doschek, G. A., \& Feldman, U. 1979, ApJ, 227, 1037

Cirtain, J. W., et al. 2013, Nature, 493, 501

Culhane, J. L., et al. 2007, Sol. Phys., 243, 19

De Pontieu, B., et al. 2011, Science, 331, 55

De Pontieu, B., McIntosh, S. W., Hansteen, V. H., \& Schrijver, C. J. 2009, ApJ, 701, L1

Del Zanna, G. 2008, A\&A, 481, L49

Del Zanna, G. 2013, A\&A, 555, A47

Del Zanna, G., \& Mason, H. E. 2003, A\&A, 406, 1089

Doschek, G. A., McKenzie, D. E., \& Warren, H. P. 2014, ApJ, 788, 26

Doschek, G. A., Warren, H. P., Mariska, J. T., Muglach, K., Culhane, J. L., Hara, H., \& Watanabe, T. 2008, ApJ, 686, 1362

Freeland, S. L., \& Handy, B. N. 1998, Sol. Phys., 182, 497

Hara, H., \& Ichimoto, K. 1999, ApJ, 513, 969

Hara, H., Watanabe, T., Harra, L. K., Culhane, J. L., \& Young, P. R. 2011, ApJ, 741, 107

Hara, H., Watanabe, T., Harra, L. K., Culhane, J. L., Young, P. R., Mariska, J. T., \& Doschek, G. A. 2008a, ApJ, 678, L67

Hara, H., Watanabe, T., Matsuzaki, K., Harra, L. K., Culhane, J. L., Cargill, P., Mariska, J. T., \& Doschek, G. A. 2008b, PASJ, 60, 275

Harra, L. K., Sakao, T., Mandrini, C. H., Hara, H., Imada, S., Young, P. R., van Driel-Gesztelyi, L., \& Baker, D. 2008, ApJ, 676, L147

Heyvaerts, J., \& Priest, E. R. 1983, A\&A, 117, 220

Imada, S., Hara, H., \& Watanabe, T. 2009, ApJ, 705, L208
Imada, S., Murakami, I., Watanabe, T., Hara, H., \& Shimizu, T. 2011, ApJ, 742,70

Ionson, J. A. 1978, ApJ, 226, 650

Kamio, S., Hara, H., Watanabe, T., Fredvik, T., \& Hansteen, V. H. 2010, Sol. Phys., 266, 209

Klimchuk, J. A. 2006, Sol. Phys., 234, 41

Ko, Y.-K., Doschek, G. A., Warren, H. P., \& Young, P. R. 2009, ApJ, 697, 1956

Korendyke, C. M., et al. 2006, Appl. Opt., 45, 8674

Kosugi, T., et al. 2007, Sol. Phys., 243, 3

Laming, J. M. 2004, ApJ, 614, 1063

Laming, J. M. 2012, ApJ, 744, 115

Lang, J., et al. 2006, Appl. Opt., 45, 8689

Linsky, J. L., Wood, B. E., Brown, A., \& Osten, R. A. 1998, ApJ, 492, 767

Mandrini, C. H., Démoulin, P., \& Klimchuk, J. A. 2000, ApJ, 530, 999

Mariska, J. T. 2013, Sol. Phys., 282, 629

McClements, K. G., Harrison, R. A., \& Alexander, D. 1991, Sol. Phys., 131, 41

O’Dwyer, B., Del Zanna, G., Mason, H. E., Sterling, A. C., Tripathi, D., \& Young, P. R. 2011, A\&A, 525, A137

Okamoto, T. J., Antolin, P., De Pontieu, B., Uitenbroek, H., Van

Doorsselaere, T., \& Yokoyama, T. 2015, ApJ, 809, 71

Parker, E. N. 1983, ApJ, 264, 642

Parker, E. N. 1988, ApJ, 330, 474

Parnell, C. E., \& De Moortel, I. 2012, Philosophical Transactions of the

Royal Society of London Series A, 370, 3217

Patsourakos, S., \& Klimchuk, J. A. 2006, ApJ, 647, 1452

Reale, F. 2010, Living Reviews in Solar Physics, 7, 5

Reale, F. 2014, Living Reviews in Solar Physics, 11, 4

Reale, F., McTiernan, J. M., \& Testa, P. 2009, ApJ, 704, L58

Reale, F., Testa, P., Klimchuk, J. A., \& Parenti, S. 2009, ApJ, 698, 756

Redfield, S., Ayres, T. R., Linsky, J. L., Ake, T. B., Dupree, A. K., Robinson,

R. D., \& Young, P. R. 2003, ApJ, 585, 993

Saba, J. L. R., \& Strong, K. T. 1991, ApJ, 375, 789

Sakao, T., et al. 2007, Science, 318, 1585

Schmelz, J. T., Saar, S. H., DeLuca, E. E., Golub, L., Kashyap, V. L., Weber,

M. A., \& Klimchuk, J. A. 2009, ApJ, 693, L131

Seely, J. F., Feldman, U., Schühle, U., Wilhelm, K., Curdt, W., \& Lemaire, P. 1997, ApJ, 484, L87

Teriaca, L., Warren, H. P., \& Curdt, W. 2012, ApJ, 754, L40

Testa, P., et al. 2013, ApJ, 770, L1

van Ballegooijen, A. A., Asgari-Targhi, M., Cranmer, S. R., \& DeLuca,

E. E. 2011, ApJ, 736, 3

Viall, N. M., \& Klimchuk, J. A. 2012, ApJ, 753, 35

Walsh, R. W., \& Ireland, J. 2003, A\&A Rev., 12, 1 

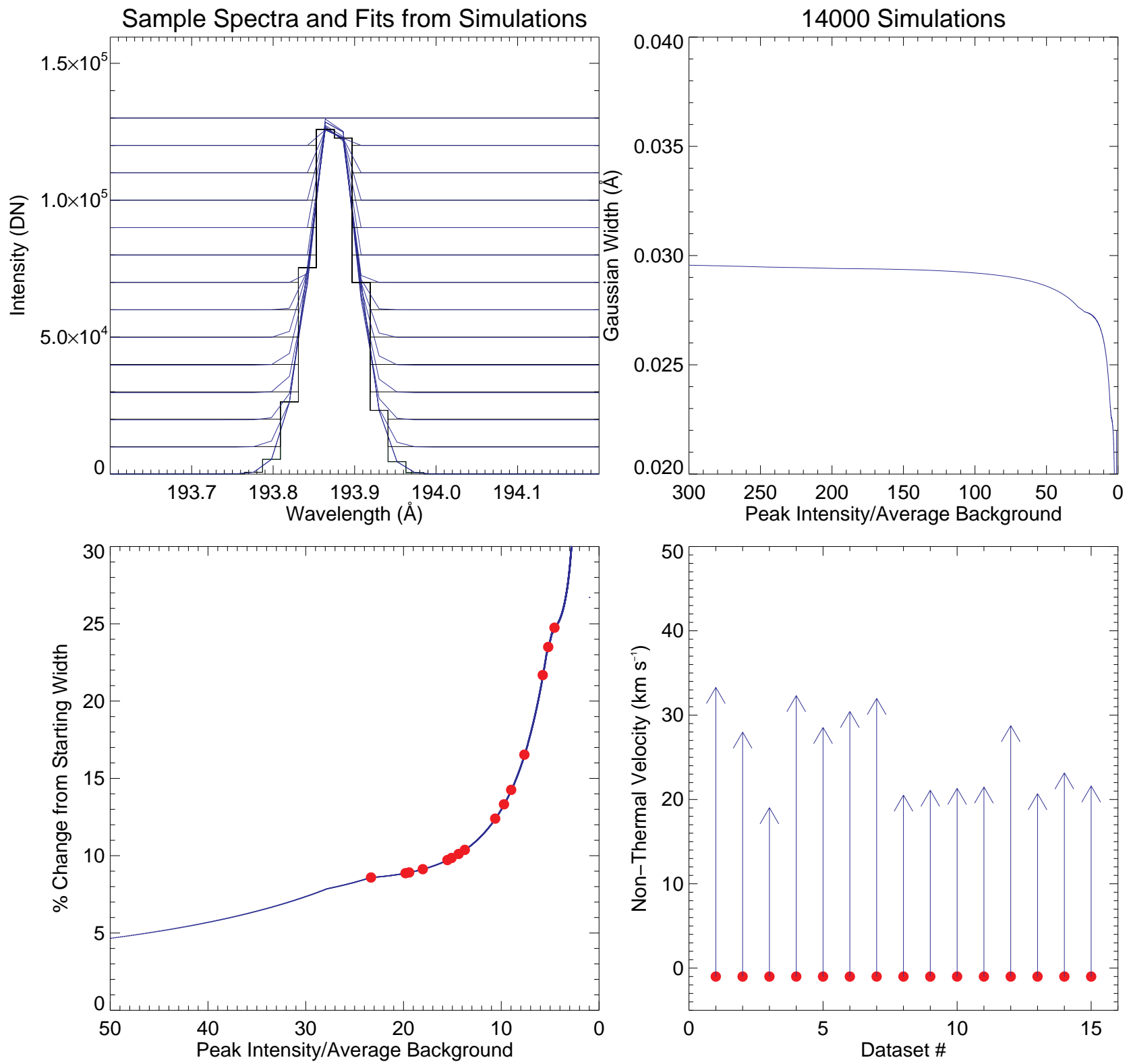

FIG. 10.- Simulations of the effect of enhanced background on the Ca XIV $193.874 \AA$ spectral line profile. Top left: a random sample of simulated profiles with increasing background levels. Gaussian fits to the profiles are overlaid in blue. Top right: The Gaussian width of the line as a function of the ratio of the peak intensity to average background level. Bottom left: the percentage change in the line profile width (compared to the initial width) as a function of the ratio of the peak intensity to average background level. The data for the $16 \mathrm{AR}$ core loops are overplotted as red dots at the actual measured values of the ratio. Bottom right: arrows showing the adjusted non-thermal velocities if the simulated correction were applied to the real data.

Warren, H. P., Ugarte-Urra, I., Doschek, G. A., Brooks, D. H., \& Williams, D. R. 2008b, ApJ, 686, L131

Warren, H. P., Ugarte-Urra, I., \& Landi, E. 2014, ApJS, 213, 11

Warren, H. P., Winebarger, A. R., \& Brooks, D. H. 2010, ApJ, 711, 228

Warren, H. P., Winebarger, A. R., \& Brooks, D. H. 2012, ApJ, 759, 141

Warren, H. P., Winebarger, A. R., Mariska, J. T., Doschek, G. A., \& Hara, H.

2008a, ApJ, 677, 1395
Wilhelm, K., et al. 1995, Sol. Phys., 162, 189

Winebarger, A. R., Warren, H. P., Schmelz, J. T., Cirtain, J., Mulu-Moore,

F., Golub, L., \& Kobayashi, K. 2012, ApJ, 746, L17

Young, P. R., 2011, EIS Software Note no. 7, Solar Software,

\$SSW/hinode/eis/doc/eis_notes

Young, P. R., et al. 2007, PASJ, 59, 857 